\title{
Geometry-dependent scaling of critical current densities for current-induced domain wall motion and transformations
}

\author{
L. Heyne, ${ }^{1}$ J. Rhensius, ${ }^{1,2}$ Y.-J. Cho, ${ }^{3}$ D. Bedau, ${ }^{1,3}$ S. Krzyk, ${ }^{1}$ C. Dette, ${ }^{1}$ H. S. Körner,${ }^{1}$ J. Fischer, ${ }^{1}$ M. Laufenberg, ${ }^{1}$ \\ D. Backes, ${ }^{1,2}$ L. J. Heyderman, ${ }^{2}$ L. Joly,${ }^{2,4}$ F. Nolting, ${ }^{2}$ G. Tatara, ${ }^{5}$ H. Kohno,${ }^{6}$ S. Seo,${ }^{3}$ U. Rüdiger, ${ }^{1}$ and M. Kläui ${ }^{1}$ \\ ${ }^{1}$ Fachbereich Physik, Universität Konstanz, 78457 Konstanz, Germany \\ ${ }^{2}$ Paul Scherrer Institut, 5232 Villigen, Switzerland \\ ${ }^{3}$ Samsung Electronics, San 14-1, Nongseo-dong, Giheung-gu, Yongin-si, Gyeonggi-do, Korea 446-711 \\ ${ }^{4}$ IPCMS, UMR 7504, 23 rue du Loess, BP 43, F-67034 Strasbourg Cedex 2, France \\ ${ }^{5}$ Department of Physics, Tokyo Metropolitan University, Hachioji, Tokyo 192-0397, Japan \\ ${ }^{6}$ Graduate School of Engineering Science, Osaka University, Toyonaka, Osaka 560-8531, Japan \\ (Received 9 May 2009; revised manuscript received 11 August 2009; published 2 November 2009)
}

\begin{abstract}
In a combined theoretical and experimental study, we investigate the critical current densities for vortex domain walls in magnetic nanowires. We systematically determine the critical current densities for continuous motion of vortex walls as a function of the wire width for different wire thicknesses and we find that the critical current density increases monotonously with decreasing wire width. Theoretically we present a mechanism that predicts a threshold current density based on wall transformations and this leads to a scaling of the critical current density $j_{c} \propto 1 /$ width. The origin of this scaling is found to be the different dependence of the spin torque energy and the vortex nucleation energy on the wire width and good agreement with the experimental observations is found.
\end{abstract}

DOI: $10.1103 /$ PhysRevB.80.184405

PACS number(s): 72.25.Ba, 75.60.Ch, 75.75.+a

Current-induced magnetization switching is a possible mechanism to realize ultra-high-density magnetic memories. Some types of such high-density memories are realized by using a switching scheme based on domain wall motion. ${ }^{1-3}$ In particular current-induced domain wall motion is of great interest, since it allows for simple designs with no need for field-generating striplines. When electric current is injected, magnetic domain walls always move in the direction of the electron flow. The domain wall motion is based on two mechanisms called the spin-transfer torque (also called adiabatic spin torque) and the effective force (sometimes called the nonadiabatic spin torque even though it originates from spin relaxation as well as nonadiabatic transport) ${ }^{4-7}$ Experiments carried out on various systems such as ferromagnetic metallic and semiconducting wires show that domain walls are driven by the spin-polarized charge current above a certain threshold value. In metals, the motion appears to be induced by the combination of the spin-transfer torque and the effective force depending on the material and magnetization configuration. ${ }^{8}$

The switching speed for a memory device based on spin torque and domain walls is directly dependent on the domain wall velocity, which therefore plays a key role. In addition to the domain wall speed, the threshold density required to drive the motion is a crucial parameter for any application, since current densities, that are too high result in heating and may cause structural damage.

The key to designing structures for applications where low-current densities are needed is a better theoretical understanding of the critical current density as a function of the wire geometry. Most experimentally observed threshold current densities in metals are believed to arise from extrinsic pinning potentials due to defects, ${ }^{7}$ but so far no quantitative argument about the origin of the threshold has been brought forward in detail. Furthermore, experimentally a systematic study of the critical current densities as a function of the geometry is lacking.

In this paper, we present a combined theoretical and experimental study of the critical current densities for vortex domain walls. We systematically determine the critical current densities for continuous motion of vortex walls as a function of wire width for different wire thicknesses and we find a monotonous dependence on the wire width.

We demonstrate that besides the extrinsic pinning there is another possible origin of the threshold current based on domain wall transformations, which is expected to be dominant for vortex walls. A theoretical analysis of this mechanism yields a dependence on the wire width that agrees with the experimental observations.

To study the critical current density in various wire geometries (12-28 nm thick, 200-1500 nm wide), we define samples on an oxidized Si substrate by electron beam lithography. The magnetic material (Permalloy: $\mathrm{Ni}_{80} \mathrm{Fe}_{20}$ ) was deposited together with a thin Au capping layer in a ultra high vacuum molecular beam epitaxy chamber (base pressure $10^{-10}$ mbar). In addition to using a lift-off process for pattern transfer, ${ }^{9}$ we use hydrogen silsesquioxane and an Ar-ion milling process, where smoother surfaces and edges are expected. In a second lithography step, Au contacts were defined for current injection.

High-resolution X-ray magnetic circular dichroism photoemission electron microscopy (XMCD-PEEM) ${ }^{10}$ is employed to directly image the magnetization configuration. ${ }^{11}$ This allows us to determine the required current density for domain wall motion by injecting current pulses with varying current density, and at the same time we can determine the domain wall spin structure and detect domain wall transformations.

In Fig. 1, we present XMCD-PEEM images of a vortex wall prior to and after pulse current injection in permalloy 

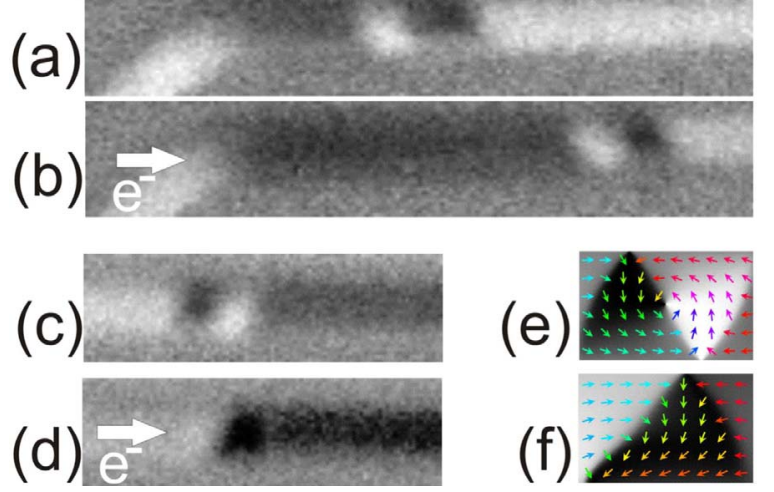

FIG. 1. (Color online) High-resolution XMCD-PEEM images of a vortex head-to-head domain wall after (a) initialization, (b) a displaced vortex wall after $25 \mu$ s pulse injection with a current density of $1.5 \times 10^{12} \mathrm{~A} / \mathrm{m}^{2}$, (c) a vortex tail-to-tail domain wall after initialization, and (d) the wall after pulse injection when it has transformed to a transverse wall spin structure. To aid visualization of the different contrast associated with the transverse and the vortex wall, simulations have been included with the arrows indicating the spin orientation [(e) and (f)].

wires (1500 nm wide, $20 \mathrm{~nm}$ thick). Figure 1(a) shows the vortex wall after initialization with a field prior to current injection. We then inject a $25 \mu$ s long current pulse with a current density of about $1.5 \times 10^{12} \mathrm{~A} / \mathrm{m}^{2}$ that is sufficiently high to induce domain wall motion. The resulting image [Fig. 1(b)] shows that the vortex wall has been displaced by $5 \mu \mathrm{m}$.

We find that the current not only displaces the domain wall but also results in domain wall transformations and that the critical current density is correlated with the onset of transformations. This highlights the major advantage of a direct imaging method that not only allows one to determine the critical current density, but also the observation of domain transformations. On repeating the experiment, while we again observe a vortex wall prior to current injection [Fig. $1(\mathrm{c})]$, on injection of a current pulse, the spin structure is observed to change [Fig. 1(d)]. The domain wall has transformed from a vortex into a transverse domain wall spin structure. Micromagnetic simulations for the two domain wall types are shown to facilitate the interpretation of the contrast [Figs. 1(e) and 1(f)].

The reason that we can see both domain wall types, is that they both constitute local energy minima for this geometry, so both spin configurations are (meta-) stable states. This is the case for geometries that are not too far from the phase boundary between transverse and vortex walls. ${ }^{11}$ As one moves away from this phase boundary, one domain wall type or the other becomes more stable and so we observe prevailing vortex or transverse walls and less often the energetically more unfavorable type. But dynamically the transformations still occur, since we do observe the domain wall type changes, from time to time.

We image domain wall displacements and transformations and determine the critical current densities for various wire dimensions. In Fig. 2, the critical current densities are shown as a function of 1/width for various thicknesses.

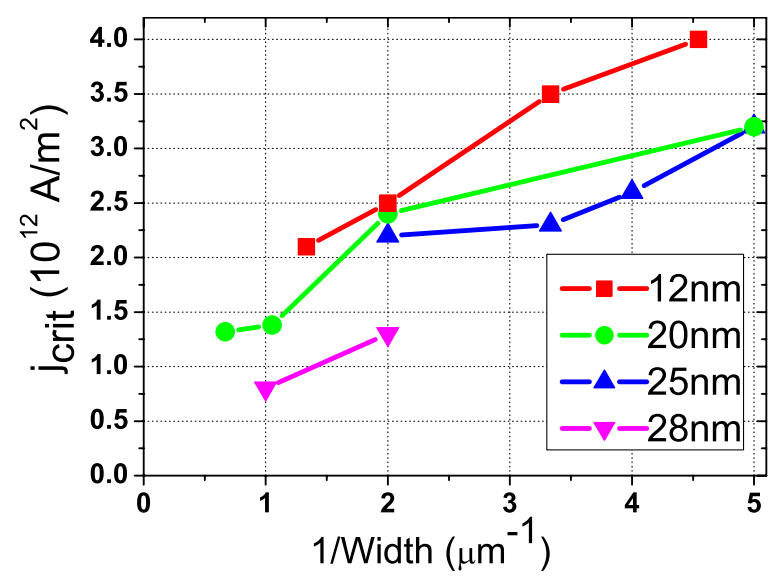

FIG. 2. (Color online) Critical current density as a function of 1/width for different thicknesses. An increase in the critical current density with decreasing wire width is seen for all thicknesses.

The relative error for the critical current densities is estimated to be around $5 \%$ for wires of the same thickness. One chip samples with different width but the same thickness are studied. Any error in the sample thickness determination therefore affects all results for this thickness simultaneously and adds an systematic relative offset to the current densities. Thus, comparing absolute current densities for samples with different thickness has to be done with care. However, since we are interested in the width dependence for fixed thicknesses this does not pose a problem for our analysis.

We observe an increase in the critical current density with decreasing wire width for all thicknesses. To comprehend this monotonic dependence, we have to understand what physically governs the threshold current density. Previously we found that the critical current densities of transverse and vortex domain walls can differ possibly due to the different pinning mechanisms at the rough wire edge and other irregularities. $^{12,13}$

To develop a simple theoretical model for the critical current density of vortex walls, we start with the mechanism of the domain wall transformation based on vortex-antivortex nucleation. We will demonstrate that this nucleation process leads to a threshold current for continuous vortex wall motion. Our theory is based on a simple analytical description of the vortex, neglecting thermal excitations and defects and we assume that the barrier necessary to flip the polarity and thus initiate the transformation governs the critical current density.

In Fig. 3, simulations of the transformation process are presented with the color code representing the out-of-plane component and the arrows indicating the in-plane magnetization. The $x$-direction is chosen to be along the wire and $y$ is the transverse direction so that the wire lies in the $x y$ plane and the vortex core points out-of-plane. Starting from a vortex wall in Fig. 3(a) the spin transfer torque results in a vortex wall motion with a velocity $v_{S} \equiv \frac{P a^{3}}{2 e S} j$ in the electron direction, where $j$ is the current density, $P$ is the spin polarization of the current, $S$ is the magnitude of the localized spin and $a$ is the lattice constant. The spin damping and effective force act as forces perpendicular to the motion, with a mag- 

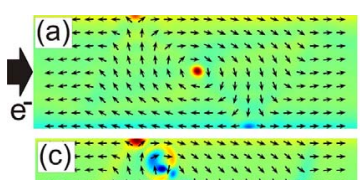

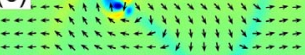

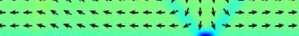

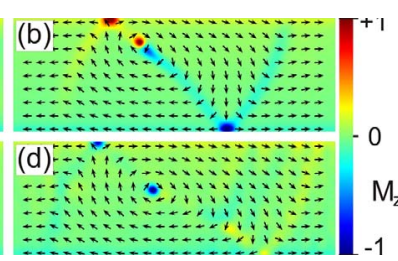

FIG. 3. (Color online) Micromagnetic simulation of the transformation process in a $300 \mathrm{~nm}$ wide and $30 \mathrm{~nm}$ thick Py wire. The color code shows the out-of-plane component (green means inplane magnetized) and the arrows indicate the in-plane magnetization direction. (a) The initial vortex wall starts to move under current injection in the direction of the electron flow $\left(j=2 \times 10^{12} \mathrm{~A} / \mathrm{m}^{2}\right)$. In addition the vortex core (red) also moves perpendicular to the electron flow toward one of the edges resulting in a distortion of the core (blue) as shown in (b). If the distortion is large enough a vortex-antivortex pair is created follow by the immediate annihilation of the old vortex with the antivortex. A snapshot of this process is shown in (c). The remaining new vortex has opposite polarity (blue) and starts moving toward the opposite edge of the wire (d).

nitude proportional to $\alpha v_{x}$ and $\beta j$, respectively $\left(v_{x}\right.$ is the vortex core velocity in the $x$-direction and $\beta$ is the nonadiabaticity parameter). ${ }^{4,13,14}$

The direction of the perpendicular vortex core motion is determined by the polarity of the vortex core (direction of the out-of-plane magnetization of the vortex core) in the wall. ${ }^{14,15}$ When the vortex core comes close to the wire edge, as shown in Fig. 3(b), it feels a repulsive force due to the increase in the stray field energy as the vortex approaches the edge. This causes a deformation of the domain wall toward a transverse wall and will eventually stop the motion. ${ }^{12}$ For the motion to proceed, the vortex core needs to flip its polarity and will then start to move toward the opposite wire edge. This domain wall propagation process requires a vortexantivortex pair to be nucleated, both having a polarization opposite to that of the original vortex core. ${ }^{16}$ The nucleated antivortex will annihilate with the original vortex, leaving a new vortex with opposite polarity. A snapshot of this mechanism is shown in Fig. 3(c), here from the out-of-plane component opposite to the original vortex that is already present in Fig. 3(c) a vortex antivortex pair is created followed by the immediate annihilation process. A vortex with opposite polarity remains that starts moving in the other direction as shown in Fig. 3(d).

The creation energy of the vortex-antivortex pair is determined by the hard axis anisotropy energy $K_{\perp}$ as: $E_{K_{\perp}} \equiv 2 \int d^{3} x \frac{1}{2} J\left|\nabla_{x} S\right|^{2} \sim 2 K_{\perp}\left(a_{v}\right)^{2} d / a^{3}$, where $a_{v}$ is the size of the vortex core, $d$ is the thickness of the system ( $J$ is the exchange coupling). This energy has to be provided by the spin-polarized current in order to secure continuous motion. Under applied current the domain wall gains spin transfer energy with decreasing distance of the vortex from the wire edge and is maximized in the limit of a transverse wall. From the Hamiltonian of the spin transfer effect: ${ }^{17}$

$$
H_{\mathrm{ST}} \equiv \int \frac{\hbar P}{2 e} \mathbf{j} \cdot \nabla \phi(1-\cos \theta) d^{3} x,
$$

the spin transfer energy of a transverse wall is estimated to be

$$
E_{\mathrm{ST}}=\pi \frac{\hbar d P}{e} j W
$$

where we have assumed a simple two dimensional wall profile (head-to-head $180^{\circ}$ domain wall and $\theta=\frac{\pi}{2}$ ). For $E_{\mathrm{ST}}>E_{K_{\perp}}$ a vortex-antivortex pair can be nucleated with the new antivortex and the original vortex annihilating each other. The remaining new vortex with the opposite polarity starts to move to the other wire edge reducing again the spin transfer energy. Thus, the threshold current density is given by

$$
j_{c}=\frac{2 e}{\pi \hbar} \frac{a_{v}^{2}}{a^{3}} \frac{K_{\perp}}{W} .
$$

This result now yields a direct dependence of the critical current density $j_{c}$ on the wire width $\mathrm{W}$ that scales with $j_{c} \propto 1 / W$. This contrasts with the case of the creation of a rigid transverse wall, where the threshold current is independent of the system size. In fact, when a transverse domain wall is created perpendicular to the current, the energy gained from the spin transfer torque is estimated to be proportional to the wire width: $\sim \frac{\hbar P}{e} j W a$, but the creation energy is also proportional to the width: $\sim K \lambda \frac{W}{a^{2}}$ ( $K$ and $\lambda$ are the easy-axis anisotropy energy and the domain wall thickness, respectively), and this results in a size-independent threshold current density $j_{c} \sim \frac{e}{\hbar a^{3}} K \lambda .^{17}$

We, thus find that the critical current density behavior strongly depends on the domain wall spin structure and that the critical current for vortex core switching depends on the geometry. The experimental observations shown in Fig. 2 qualitatively agree with the prediction of the $j_{c} \propto 1 / W$ scaling for vortex walls in that the critical current density increases with decreasing wire width for all thicknesses. To obtain a more quantitative comparison, we can estimate the magnitude of the threshold current density as follows: Noting that the vortex core radius is given by the ratio of the exchange and anisotropy energy as $a_{v} \sim \sqrt{J / K_{\perp}}$, the threshold current [Eq. (3)] is written as $j_{c} \sim \frac{e}{\hbar} \frac{1}{a W} \frac{J}{a^{2}}$. For $3 \mathrm{~d}$ ferromagnets generic values are $J / a^{2} \sim 10^{-19} \mathrm{~J}$ (per site), and $a \sim 2.5 \times 10^{-10} \mathrm{~m}$ and with this we obtain $j_{c} \sim 2 \times 10^{15} \frac{a}{W} \mathrm{~A} / \mathrm{m}^{2}$. Therefore, for a width of $500 \mathrm{~nm}$, $j_{c} \sim 10^{12} \mathrm{~A} / \mathrm{m}^{2}$, which agrees well within the error with the critical current densities obtained in the experiments. Given the large number of (reasonable) assumptions in the theoretical model (no thermal excitations, simple analytical description of the vortex structure, no influence of defects, scaling of the vortex size with the wire width, etc.) a closer quantitative agreement can not be expected.

The experimental results further suggest that in reality a combination of different depinning mechanisms prevail since the obtained curves are best described by a linear function with an small offset $j_{o}: j_{c} \propto 1 /$ width $+j_{o}$. The slope seems not to depend on the thickness in agreement with our theory. The nonzero offset $j_{o}$ could be explained by extrinsic pinning that also contributes to the critical current density. 
In conclusion, we have determined experimentally and theoretically the dependence of the threshold current density for continuous vortex wall motion. Theoretically we find a scaling of $j_{c} \propto 1 /$ width and this size dependence arises from the fact that the vortex-antivortex creation energy is independent of the wire width, while the energy gain depends on the wall size, which scales with the wire width. Whereas for rigid transverse walls the threshold current density is independent of the geometry.

Experimentally we have imaged vortex domain wall spin structures, their displacement and their transformations for various geometries, and we find an increase of the critical current density with decreasing wire width for all thicknesses, which is in agreement with our theoretical model of periodic domain wall transformations.

\section{ACKNOWLEDGMENTS}

G.T. acknowledges Grant-in-Aid for Scientific Research on Priority Areas for financial support. M.K. acknowledges the German Science Foundation (Grants No. DFG SFB 513, No. SFB 767, and No. KL1811), the European Research Council Starting Independent Researcher Grant (No. ERC2007-Stg 208162) scheme, EU RTN SPINSWITCH (MRTNCT-2006-035327) and the Samsung Advanced Institute of Technology. We thank A. Locatelli at ELETTRA and $\mathrm{S}$. Dhesi at DIAMOND for help with instrumentational development. Part of this work was carried out at the Swiss Light Source, Paul Scherrer Institut.
${ }^{1}$ S. S. P. Parkin, M. Hayashi, and L. Thomas, Science 320, 190 (2008).

${ }^{2}$ R. Cowburn, D. Petit, D. Read, and O. Petracic, Patent No. WO 132174A1 (2007).

${ }^{3}$ D. Ilgaz, M. Kläui, L. Heyne, O. Boulle, F. Zinser, S. Krzyk, M. Fonin, U. Rüdiger, D. Backes, and L. J. Heyderman, Appl. Phys. Lett. 93, 132503 (2008).

${ }^{4}$ A. Thiaville, Y. Nakatani, J. Miltat, and Y. Suzuki, Europhys. Lett. 69, 990 (2005).

${ }^{5}$ S. Zhang and Z. Li, Phys. Rev. Lett. 93, 127204 (2004).

${ }^{6}$ G. Tatara and H. Kohno, Phys. Rev. Lett. 92, 086601 (2004).

${ }^{7}$ G. Tatara, H. Kohno, and J. Shibata, Phys. Rep. 468, 213 (2008).

${ }^{8}$ O. Boulle, J. Kimling, P. Warnicke, M. Kläui, U. Rüdiger, G. Malinowski, H. J. M. Swagten, B. Koopmans, C. Ulysse, and G. Faini, Phys. Rev. Lett. 101, 216601 (2008).

${ }^{9}$ L. J. Heyderman, M. Kläui, B. Nöhammer, C. A. F. Vaz, J. A. C. Bland, and C. David, Microelectron. Eng. 73-74, 780 (2004).

${ }^{10}$ J. Stöhr, Y. Wu, B. D. Hermsmeier, M. G. Samant, G. R. Harp, S. Koranda, D. Dunham, and B. P. Tonner, Science 259, 658
(1993).

${ }^{11}$ M. Kläui, J. Phys.: Condens. Matter 20, 313001 (2008).

${ }^{12}$ M. Kläui, P. O. Jubert, R. Allenspach, A. Bischof, J. A. C. Bland, G. Faini, U. Rüdiger, C. A. F. Vaz, L. Vila, and C. Vouille, Phys. Rev. Lett. 95, 026601 (2005).

${ }^{13}$ L. Heyne, M. Kläui, D. Backes, T. A. Moore, S. Krzyk, U. Rüdiger, L. J. Heyderman, A. Fraile Rodriguez, F. Nolting, T. O. Mentes, M. A. Nino, A. Locatelli, K. Kirsch, and R. Mattheis, Phys. Rev. Lett. 100, 066603 (2008).

${ }^{14}$ J. Shibata, Y. Nakatani, G. Tatara, H. Kohno, and Y. Otani, Phys. Rev. B 73, 020403(R) (2006).

${ }^{15}$ Y. Nakatani, J. Shibata, G. Tatara, H. Kohno, A. Thiaville, and J. Miltat, Phys. Rev. B 77, 014439 (2008).

${ }^{16}$ B. Van Waeyenberge, A. Puzic, H. Stoll, K. W. Chou, T. Tyliszczak, R. Hertel, M. Fähnle, H. Brück, K. Rott, G. Reiss, I. Neudecker, D. Weiss, C. H. Back, and G. Schütz, Nature (London) 444, 461 (2006).

${ }^{17}$ J. Shibata, G. Tatara, and H. Kohno, Phys. Rev. Lett. 94, 076601 (2005). 